\title{
Pharmacokinetics, Antiviral Activity, and Safety of Rilpivirine in Pregnant Women with HIV-1 Infection: Results of a Phase 3b, Multicenter, Open-Label Study
}

\author{
Olayemi Osiyemi · Salih Yasin • Carmen Zorrilla - Ceyhun Bicer • \\ Vera Hillewaert · Kimberley Brown · Herta M. Crauwels
}

Received: November 8, 2017 / Published online: January 15, 2018

(C) The Author(s) 2018. This article is an open access publication

\begin{abstract}
Introduction: Physiologic changes during pregnancy may impact the pharmacokinetics of drugs. In addition, efficacy and safety/tolerability concerns have been identified for some antiretroviral agents.

Methods: Human immunodeficiency virus (HIV)-1-infected pregnant women (1826 weeks gestation) receiving the non-nucleoside reverse transcriptase inhibitor rilpivirine $25 \mathrm{mg}$ once daily were enrolled in this phase $3 \mathrm{~b}$,

Enhanced content To view enhanced content for this article go to http://www.medengine.com/Redeem/ DD1DF06034FBB700.
\end{abstract}

O. Osiyemi $(\bowtie)$

Triple O Research Institute PA, West Palm Beach, FL, USA

e-mail: drtripleo@aol.com

S. Yasin

University of Miami School of Medicine, Miami, FL, USA

C. Zorrilla

University of Puerto Rico School of Medicine, San Juan, Puerto Rico

C. Bicer

BICER Consulting \& Research, Antwerp, Belgium

V. Hillewaert · H. M. Crauwels

Janssen Research \& Development, Beerse, Belgium

K. Brown

Janssen Scientific Affairs, LLC, Titusville, NJ, USA open-label study examining the impact of pregnancy on the pharmacokinetics of rilpivirine when it is given in combination with other antiretroviral agents. Blood samples (collected over the 24-h dosing interval) to assess total and unbound rilpivirine plasma concentrations were obtained during the second and third trimesters (24-28 and 34-38 weeks gestation, respectively) and 6-12 weeks postpartum. Pharmacokinetic parameters were derived using noncompartmental analysis and compared (pregnancy versus postpartum) using linear mixed effects modeling. Antiviral and immunologic response and safety were assessed. Results: Nineteen women were enrolled; 15 had evaluable pharmacokinetic results. Total rilpivirine exposure was 29-31\% lower during pregnancy versus postpartum; differences were less pronounced for unbound (pharmacodynamically active) rilpivirine. At study entry, $12 / 19(63.2 \%)$ women were virologically suppressed; 10/12 (83.3\%) women were suppressed at the postpartum visit. Twelve infants were born to the 12 women who completed the study (7 discontinued); no perinatal viral transmission was observed among 10 infants with available data. Rilpivirine was generally safe and well tolerated in women and infants exposed in utero.

Conclusion: Despite decreased rilpivirine exposure during pregnancy, treatment was effective in preventing mother-to-child transmission and suppressing HIV-1 RNA in pregnant women. 
Results suggest that rilpivirine $25 \mathrm{mg}$ once daily, as part of individualized combination antiretroviral therapy, may be an appropriate option for HIV-1-infected pregnant women.

Trial Registration: ClinicalTrials.gov Identifier, NCT00855335.

Keywords: HIV; Pharmacokinetics; Pregnancy; Rilpivirine

\section{INTRODUCTION}

Combination antiretroviral therapy (cART) is recommended for pregnant women with human immunodeficiency virus (HIV)-1 infection to suppress virus replication and reduce the risk of perinatal virus transmission $[1,2]$. Because women experience physiologic changes during pregnancy, it is important to assess the potential impact on the pharmacokinetic parameters of antiretroviral (ARV) agents [3]. Previous studies have demonstrated reduced exposure to protease inhibitors during pregnancy [4-6], while exposures to the non-nucleoside reverse transcriptase inhibitors (NNRTIs) nevirapine, efavirenz, etravirine, and rilpivirine have been reported to be reduced, largely similar, or even increased compared with postpartum [7-12].

Rilpivirine is approved for the treatment of HIV-1 infection, as part of cART, in treatmentnaïve individuals and, in most countries, is restricted to those with HIV-1 RNA $\leq 100,000$ copies/mL [13]. The efficacy and safety of rilpivirine in nonpregnant, treatmentnaïve adults with HIV-1 infection have been demonstrated in 2 randomized, double-blinded, active-controlled, phase 3 studies (ECHO and THRIVE) $[14,15]$. Other studies have demonstrated that virologically suppressed, HIV-1-infected subjects maintained virologic suppression after switching to a rilpivirinecontaining complete regimen [either emtricitabine/rilpivirine/tenofovir disoproxil fumarate (TDF) or emtricitabine/rilpivirine/tenofovir alafenamide (TAF)] [16-18]. Current US Department of Health and Human Services (DHHS) guidelines [1] recommend the use of rilpivirine in combination with emtricitabine and TDF, or emtricitabine and TAF, in certain clinical situations in nonpregnant, treatmentnaïve adults with pretreatment HIV-1 RNA $<100,000$ copies/mL and a CD4+ count of $>200$ cells $/ \mathrm{mm}^{3}$.

Rilpivirine is currently recommended for the treatment of HIV-1-infected pregnant women in US Perinatal Guidelines (as an alternative agent), and guidelines from the European AIDS Clinical Society (EACS) support its continued use during pregnancy [2, 19]. Animal studies have shown no evidence of teratogenicity with rilpivirine and, according to the Antiretroviral Pregnancy Registry, for rilpivirine, "sufficient numbers of first trimester exposures have been monitored to detect at least a twofold increase in risk of overall birth defects. No such increases have been detected to date" [20]. Clinical studies from the PANNA Network and IMPAACT P1026s have demonstrated reduced exposure to rilpivirine during pregnancy, but no perinatal transmission has been observed [11-13, 20].

In the present study, the pharmacokinetic parameters of several ARV agents, including rilpivirine, were evaluated during pregnancy and postpartum. Antiviral activity, safety/tolerability, and infant outcomes were also assessed. Results from the rilpivirine treatment arm are reported here; results from other treatment arms have been reported previously $[5,6,9]$.

\section{METHODS}

\section{Study Design and Treatment}

HIV-1-infected pregnant women at least 18 years of age were enrolled in this phase $3 \mathrm{~b}$, multicenter, open-label study to assess the influence of pregnancy on the pharmacokinetic parameters of ARV agents, including darunavir boosted by ritonavir [twice-daily (bid) and oncedaily (qd) regimens] or cobicistat (qd), etravirine (bid), and rilpivirine (qd), as part of cART (ClinicalTrials.gov Identifier: NCT00855335).

Treatment in the rilpivirine arm included rilpivirine $25 \mathrm{mg}$ qd, in combination with other ARVs, administered as either a combination of separate agents EDURANT $^{\circledR}$; Janssen 
Therapeutics) or as part of the complete regimen rilpivirine/emtricitabine/TDF (COMPLERA $^{\circledR}$; Gilead Sciences) [13, 21]. Rilpivirine was dispensed to subjects under the supervision of the investigator, a qualified member of the investigational staff, or by a hospital/clinic pharmacist. Rilpivirine was taken with a meal. Eligible subjects were HIV-1-infected women in the second trimester of pregnancy (18-26 weeks gestation) and receiving rilpivirine $25 \mathrm{mg}$ qd as part of their ARV regimen at the time of study entry. For women receiving rilpivirine as part of their first line of therapy (as a single agent in combination with other ARVs or as part of the complete regimen rilpivirine/emtricitabine/ TDF), they must have had pretreatment HIV-1 RNA $<100,000$ copies/mL, no evidence of specific NNRTI resistance-associated mutations (RAMs; K101E, K101P, E138A, E138G, E138K, E138R, E138Q, V179L, Y181C, Y181I, Y181V, H221Y, F227C, M230I or M230L, or the combination of K103N and L100I), and been using rilpivirine in combination with two active nucleos(t)ides. For women receiving rilpivirine as part of the complete regimen rilpivirine/ emtricitabine/TDF and treatment-experienced (i.e., switched from another ARV regimen), they must have had no history of virologic failure, been virologically suppressed for $\geq 6$ months prior to switching to rilpivirine/emtricitabine/ TDF, been on their first or second ARV regimen prior to switching to rilpivirine/emtricitabine/ TDF, and have no current or past history of resistance to rilpivirine, emtricitabine, or TDF. Additional eligibility criteria included a normal obstetrical exam within 2 weeks of the screening visit, a normal fetal ultrasound, receiving care for pregnancy and HIV management from an obstetrician and/or primary HIV healthcare provider and agreed to continue doing so for the duration of the study, willing to remain on rilpivirine and a background regimen for the duration of the study (including 12 weeks postpartum), and able to comply with the protocol requirements.

Exclusion criteria included active acquired immunodeficiency syndrome (AIDS)-defining illness (except stable, cutaneous Kaposi sarcoma or wasting syndrome caused by HIV infection); presence of newly diagnosed HIV-related opportunistic infection or any medical condition requiring acute therapy; use of certain concomitant medications; use of disallowed medication per the current prescribing information (as appropriate) for rilpivirine and the ARV background regimen; use of an investigational agent within 90 days prior to screening; any current obstetrical complication; any known fetal anomaly; uncontrolled diabetes mellitus type 1 or 2 , or gestational diabetes; untreated hypothyroidism or hyperthyroidism; certain hepatic abnormalities; certain laboratory abnormalities; neurological condition requiring medication; current alcohol or recreational drug use; and any condition that could compromise the subject's safety or adherence to the protocol.

Adherence to study medications was assessed based on 4-day recall, pill counts, maintenance of adequate medication dispensing, and return records of all medications provided.

The primary objective of this analysis was to compare rilpivirine pharmacokinetic parameters during the second and third trimesters of pregnancy to those postpartum. Secondary objectives were to evaluate antiviral activity, safety, and tolerability of rilpivirine-based ARV regimens during pregnancy and postpartum; to compare rilpivirine concentrations between plasma and cord blood samples at the time of delivery; and to assess outcomes for infants of women treated with rilpivirine during pregnancy.

All subjects provided written informed consent to participate in this study. The study was conducted in accordance with the ethical principles that have their origin in the 1964 Declaration of Helsinki, and its later amendments, and is consistent with Good Clinical Practices and applicable regulatory requirements. The study protocol and amendments were reviewed by an independent ethics committee or institutional review board.

\section{Pharmacokinetic Evaluations}

Blood samples were collected over the 24-h dosing interval to assess the plasma pharmacokinetics of total and unbound rilpivirine. 
Pharmacokinetic evaluations occurred at clinic visits during the second and third trimesters of pregnancy (24-28 and 34-38 weeks gestation, respectively) and 6-12 weeks postpartum; during these visits, study staff observed subjects' intake of rilpivirine. Matching cord blood and maternal plasma samples were taken at the intrapartum visit (when feasible). Plasma concentrations of rilpivirine were determined using a validated, specific, and sensitive liquid chromatography mass spectrometry/mass spectrometry (LC-MS/MS) method. The fraction of unbound rilpivirine was determined via separation through dialysis of pooled (per subject and per pharmacokinetic visit) plasma samples and LC-MS/MS of the fractions. Pharmacokinetic parameters were derived using noncompartmental analysis (model 200, extravascular input, plasma data; Phoenix ${ }^{\mathrm{TM}}$ WinNonlin $^{\circledR}$, v.6.2.1; Tripos LP, St. Louis, MO, USA) and included area under the plasma concentration versus time curve from time of administration to $24 \mathrm{~h}$ postdose $\left(\mathrm{AUC}_{24 \mathrm{~h}}\right)$, maximum plasma concentration $\left(C_{\max }\right)$, minimum plasma concentration $\left(C_{\mathrm{min}}\right)$, observed plasma concentration prior to the beginning of a dosing interval $\left(C_{0 \mathrm{~h}}\right)$, and time to reach the maximum plasma concentration $\left(t_{\max }\right)$.

\section{Antiviral Activity and Safety/Tolerability}

Antiviral response (defined as HIV-1 RNA $<50$ copies/mL), immunologic response, and safety/tolerability were evaluated at each study visit, beginning with the baseline visit at 1826 weeks gestation. Immunologic response was reported as $\mathrm{CD} 4+$ percentage, in lieu of absolute $\mathrm{CD} 4+$ count, because CD4+ percentage has been reported to be more stable in HIV-1-negative women during pregnancy compared with the 12-week postdelivery period [22]. Laboratory parameters included albumin and $\alpha_{1}$-acid glycoprotein; concentrations of these plasma proteins are typically reduced during pregnancy due to hemodilution [23] and rilpivirine is highly bound to plasma proteins [13].

\section{Statistical Analyses}

Rilpivirine pharmacokinetic parameters (total and unbound) were summarized per exposure period [second and third trimesters (tests) and postpartum (reference)] and compared between pregnancy and postpartum using linear mixed effects modeling. Efficacy and safety data were summarized using descriptive statistics; no comparisons across exposure periods were performed.

\section{RESULTS}

\section{Subject Disposition}

A total of 19 women were enrolled in the rilpivirine arm of the study and all received rilpivirine $25 \mathrm{mg}$ qd (intent-to-treat population). Fifteen of the 19 women (79\%) had $\geq 1$ pharmacokinetic sample taken, and were thus included in the pharmacokinetic population. Evaluable pharmacokinetic results were available for 15, 13, and 11 women for the second trimester, third trimester, and postpartum visits, respectively. Twelve of the 19 women (63\%) completed the study; reasons for discontinuation of the remaining 7 women included: pregnancy terminated $(n=1)$, did not fulfill all inclusion/exclusion criteria $[n=2$ (due to exclusionary laboratory result and preexisting rilpivirine RAMs)], lost to follow-up $(n=1)$, noncompliant $(n=1)$, withdrew consent $(n=1)$, and other $[n=1$ (woman had suspected virologic failure, but was suppressed at the withdrawal visit)]. All 12 women who completed the study gave birth to 1 infant each; HIV-1 infection data were available for 10 of these infants.

\section{Subject Population}

The median (range) age of the women at screening was 26 (21-36) years, 17 (90\%) women were black or African American, 12 (63\%) had HIV-1 RNA < 50 copies $/ \mathrm{mL}$, the median (range) HIV-1 RNA level was 1.69 (1.3-3.4) $\log _{10}$ copies/mL, the median (range) 
CD4+ count was 427.0 (16-1296) cells $/ \mathrm{mm}^{3}$, and the median (range) time since conception was 158 (137-190) days (Table 1). Per the inclusion criteria, all women were taking rilpivirine $25 \mathrm{mg}$ qd at baseline; 3 (16\%) women used rilpivirine as a single agent in combination

Table 1 Baseline demographic and disease characteristics

$\begin{array}{ll}\text { Baseline characteristics } & n=19\end{array}$

\section{Demographic characteristics}

Age at screening, median (range) (years)

Race/ethnicity, $n$ (\%)

White

Black or African American

Hispanic

BMI, median (range) $\left(\mathrm{kg} / \mathrm{m}^{2}\right)$

First pregnancy, $n$ (\%)

No

Yes

Time since conception, median (range) (days)

$158(137-190)$

\section{Disease characteristics}

Known duration of HIV infection, median (range) (years)

$0.5(0.2-12.8)$

HIV-1 RNA at baseline (copies/mL), $n$ (\%)

$$
<50
$$

50 to $<400$

$4(21)$

400 to $<1000$

$1(5)$

$\geq 1000$

CD4+ count at baseline (cells $\left./ \mathrm{mm}^{3}\right), n$ (\%)

$$
<50
$$

$$
50 \text { to }<100
$$

0

100 to $<200$

200 to $<350$

$\geq 350$

Combination ARVs used with rilpivirine at baseline, $n(\%)^{a}$

Emtricitabine + TDF

Emtricitabine $+\mathrm{TDF}+$ zidovudine

Lamivudine + zidovudine

$1(5)$

$B M I$ body mass index, $H I V$ human immunodeficiency virus, $A R V$ antiretroviral, $T D F$ tenofovir disoproxil fumarate

a Sixteen women used the complete regimen rilpivirine/emtricitabine/TDF and 3 women used rilpivirine as a single agent in combination with other ARVs 
with other ARVs and 16 (84\%) women used the complete regimen rilpivirine/emtricitabine/ TDF. Among the 16 women who used the complete regimen, 3 (19\%) were treatmentexperienced and virologically suppressed (without previous virologic failure) when they switched to rilpivirine/emtricitabine/TDF, and 13 $(81 \%)$ used the complete regimen as their first line of therapy. Among all women, background regimens included emtricitabine and TDF [ $n=10(53 \%)]$; emtricitabine, TDF, and zidovudine $[n=8(42 \%)]$; and lamivudine and zidovudine $[n=1(5 \%)]$. All $9(47 \%)$ women who were using zidovudine were enrolled at a single study site and started cART with oral zidovudine as a standard-of-care practice (i.e., zidovudine was not added to the regimen due to suspected virologic failure). The mean (standard error) duration of rilpivirine intake in the study was 17.7 (2.1) weeks [11.8 (1.2) weeks prebirth and $9.2(0.5)$ weeks postbirth]. The mean (standard error) percentage of rilpivirine doses reported to be taken in the 4 days preceding a visit ranged from $95 \%$ (5.0) to $100 \%$ throughout the study.

\section{Pharmacokinetics}

Total rilpivirine plasma concentrations over the entire 24-h dosing interval were lower during pregnancy than postpartum, and comparable between the second and third trimesters of pregnancy (Fig. 1). Correspondingly, mean values for total rilpivirine $C_{0 \mathrm{~h}}, C_{\min }, C_{\max }$, and $\mathrm{AUC}_{24 \mathrm{~h}}$ were similar during the second and third trimesters of pregnancy, and these values were lower than those during the postpartum period (Table 2). Compared with postpartum, total rilpivirine $\mathrm{AUC}_{24 \mathrm{~h}}$ was $29 \%$ and $31 \%$ lower during the second and third trimesters of pregnancy, respectively, and $C_{\max }$ was $21 \%$ and $20 \%$ lower during the second and third trimesters of pregnancy, respectively. The decrease in unbound rilpivirine exposure during pregnancy was less pronounced than for total rilpivirine. Compared with postpartum, unbound rilpivirine $\mathrm{AUC}_{24 \mathrm{~h}}$ was $25 \%$ and $22 \%$ lower during the second and third trimesters of pregnancy, respectively, and $C_{\max }$ was $15 \%$ and $10 \%$ lower during the second and third trimesters of

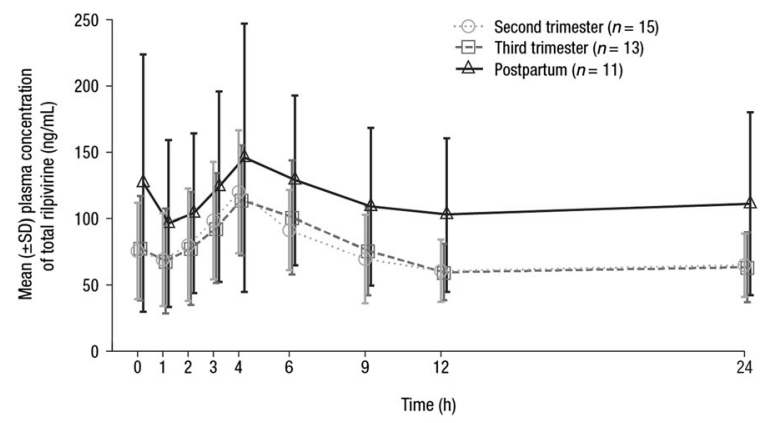

Fig. 1 Mean $( \pm S D)$ plasma concentration-time profiles of total rilpivirine during pregnancy and postpartum over the 24-h dosing interval. SD standard deviation

pregnancy, respectively. Median $t_{\max }$ was $4.00 \mathrm{~h}$ at all 3 time points during pregnancy and postpartum.

For $C_{\min }$, the statistical comparisons of total and unbound rilpivirine values during pregnancy versus postpartum were carried out both without and with 2 values that were below the limit of quantification (BLQ), which is indicative of nonadherence; 1 woman had a $C_{\text {min }}$ value BLQ at the second trimester visit (reported adherence was $75 \%$ in the 4 days prior to the visit) and 1 woman had a $C_{\min }$ value BLQ at the postpartum visit (reported adherence was 100\% in the 4 days prior to the visit). Excluding the $C_{\text {min }}$ values that were $\mathrm{BLQ}$, compared with postpartum, total rilpivirine $C_{\min }$ was $35 \%$ and $42 \%$ lower during the second and third trimesters of pregnancy, respectively; unbound $C_{\min }$ was $32 \%$ and $36 \%$ lower during the second and third trimesters of pregnancy, respectively (Table 2). Including the $C_{\min }$ values that were BLQ, compared with postpartum, the $90 \%$ confidence intervals around the least squares mean ratios were very wide; total rilpivirine $C_{\min }$ was $24 \%$ and $4 \%$ lower during the second and third trimesters of pregnancy, respectively, and unbound $C_{\min }$ was $17 \%$ lower and $12 \%$ higher during the second and third trimesters of pregnancy, respectively.

Individual cord/maternal plasma ratios of total rilpivirine on the day of delivery were analyzed in 8 women; the median ratio was 0.55 (range: 0.43-0.98). 


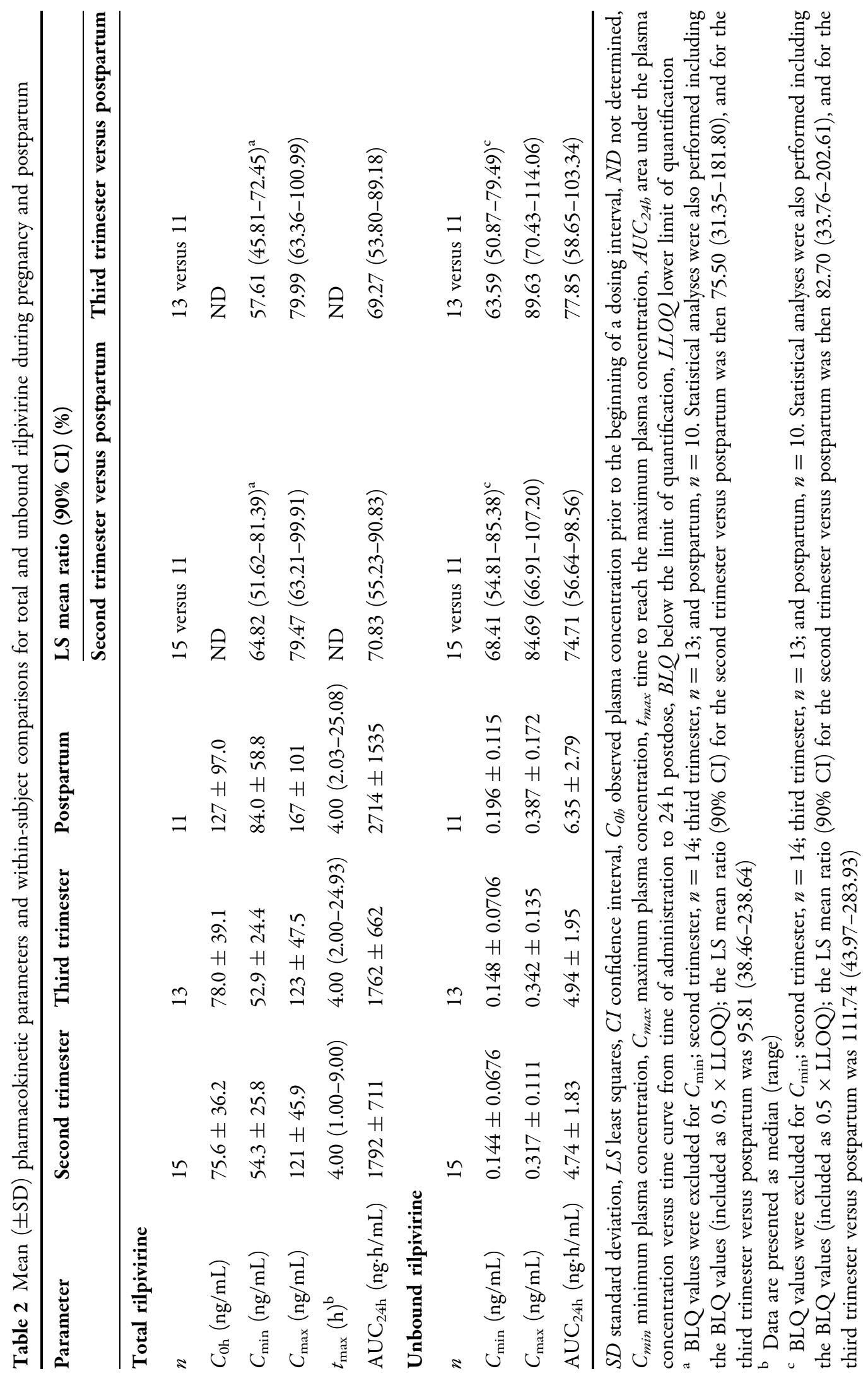




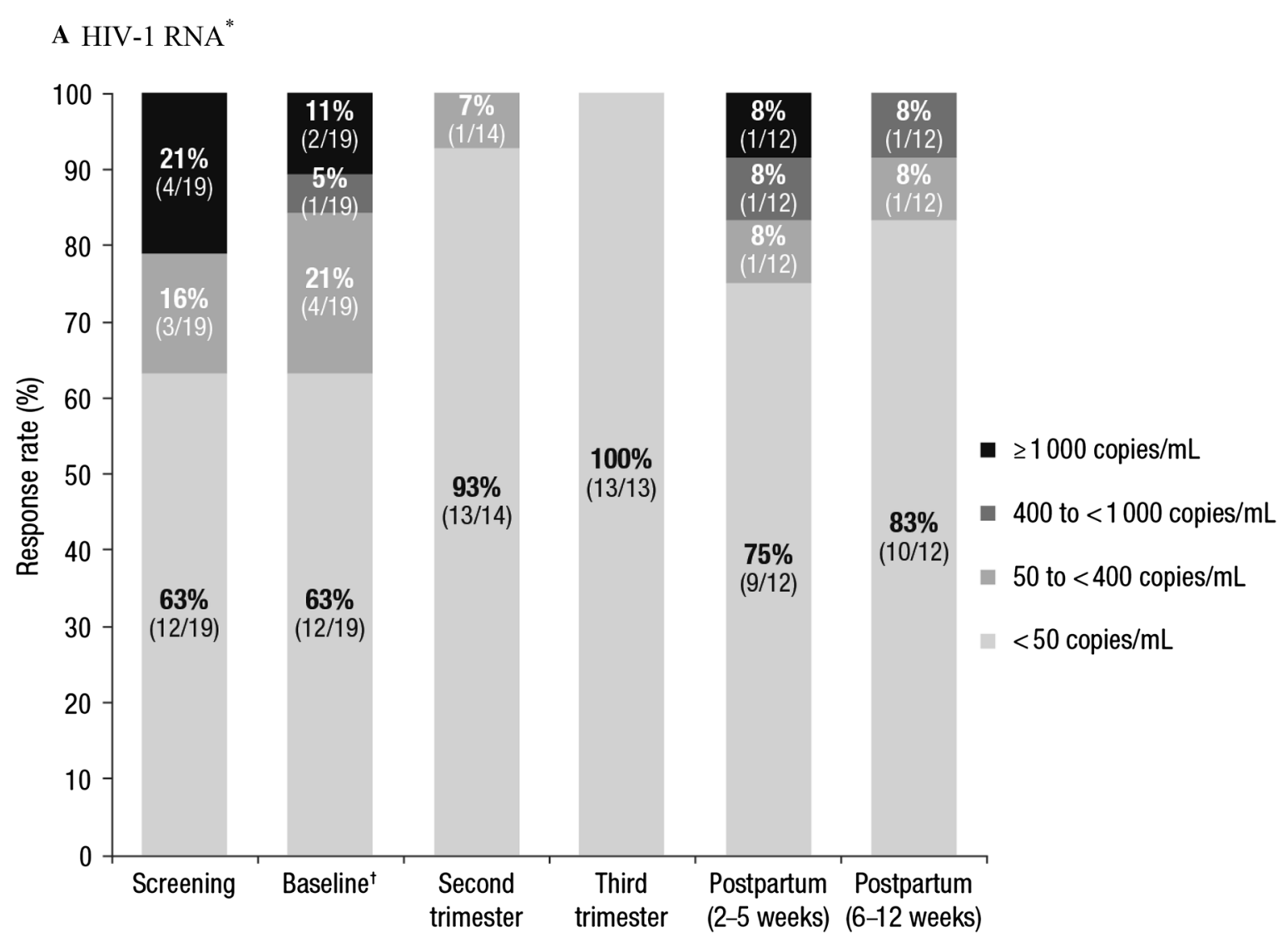

B CD4+ percentage

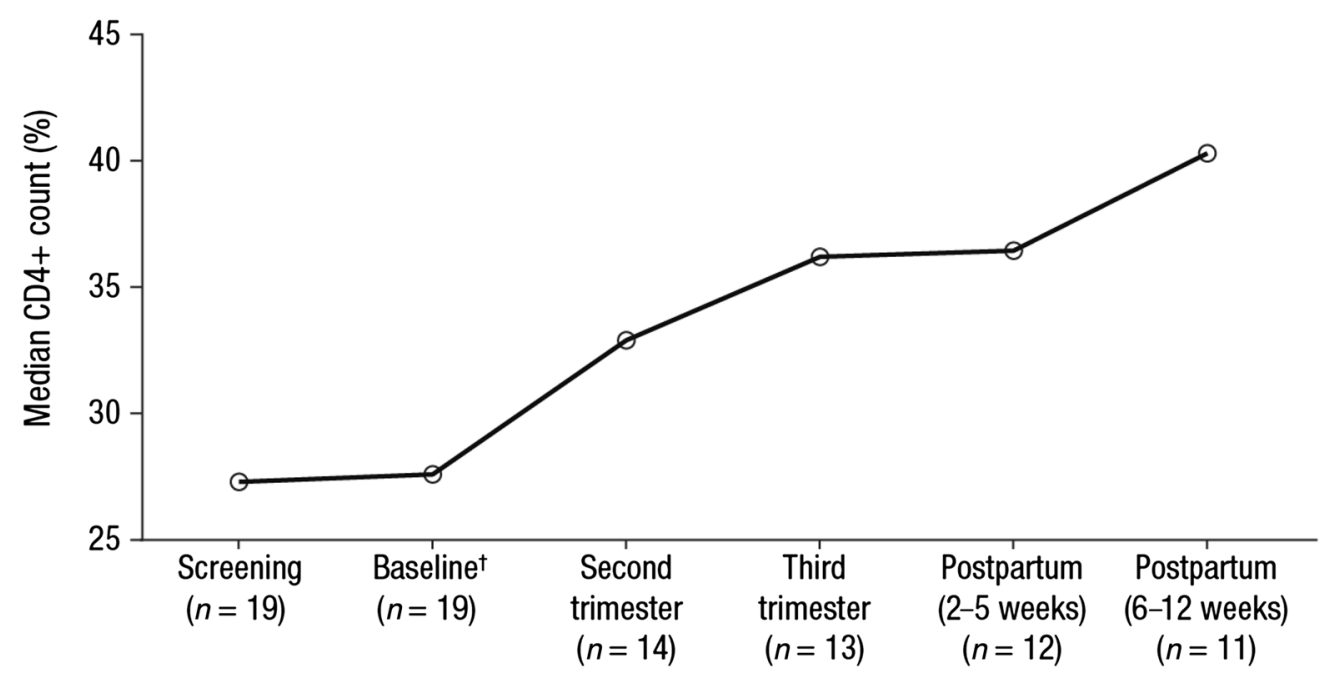

Fig. 2 Antiviral activity over time, as assessed by a HIV-1 $\mathrm{RNA}^{*}$ and $\mathbf{b} \mathrm{CD} 4+$ percentage during pregnancy and postpartum. $H I V-1$ human immunodeficiency virus-1, qd once daily, $A R V$ antiretroviral. *For each time point, percentages may not total $100 \%$ due to rounding. ${ }^{\dagger}$ The baseline visit occurred at $18-26$ weeks gestation; per the inclusion criteria, eligible subjects were receiving rilpivirine $25 \mathrm{mg}$ qd as part of their ARV regimen at the time of study entry 


\section{Efficacy}

Among the 10 infants with available data, no perinatal viral transmission was observed.

At baseline, 12 of 19 (63\%) women were virologically suppressed. Viral suppression was reached or maintained during the study in 13 of 14 (93\%) women with available data at the second trimester visit, 13 of $13(100 \%)$ at the third trimester visit, and 10 of $12(83 \%)$ at the end-of-study visit (6-12 weeks postpartum visit; Fig. 2a). Though the sample size was small, the addition of zidovudine to the background regimen did not appear to have an impact on virologic suppression data; of the 10 women who were suppressed at study completion, 3 used zidovudine in their background regimen and 7 did not use zidovudine. For the 2 women who were not suppressed at the end of study visit, both used a background regimen of emtricitabine, TDF, and zidovudine. One of these women was suppressed at baseline and all visits during pregnancy, but had an HIV-1 RNA level of 9640 copies/mL at the 2-5 weeks postpartum visit and an HIV-1 RNA level of 50 copies/mL at the 6-12 weeks postpartum visit; this woman did not meet the criteria for virologic failure. The other woman who was not suppressed at the end of study visit had virologic failure postpartum (i.e., 2 consecutive HIV-1 RNA measurements of $>200$ copies $/ \mathrm{mL}$ after reaching HIV-1 RNA $<50$ copies/mL) and suspected nonadherence postpartum (at the 6-12 weeks postpartum visit, the rilpivirine predose concentration was BLQ, while it was 155 and $131 \mathrm{ng} / \mathrm{mL}$ at the second and third trimester visits, respectively).

As noted previously, 1 woman withdrew from the study due to suspected virologic failure (background regimen: emtricitabine and TDF). Virologic failure was suspected due to HIV-1 RNA levels that did not decrease by $\geq 0.5 \log$ 4 weeks after baseline. Moreover, the rilpivirine predose concentration at the second trimester visit was BLQ, and thus indicative of nonadherence. This woman was subsequently suppressed at the time of withdrawal with an HIV-1 RNA level of 36 copies/mL. For the 2 women with (suspected) virologic failure, no resistance test results were available at the time of failure.
Median CD4+ percentage, which is reported in lieu of absolute CD4+ count (see "Methods"), increased over time (Fig. 2b).

\section{Safety/Tolerability}

Nine of 19 women (47\%) experienced $\geq 1$ adverse event (AE); none of the AEs were considered by the investigator to be at least possibly related to the study medication and none led to study discontinuation (Table 3). The most common AEs (occurring in $>1$ woman) were chorioamnionitis $[n=3(16 \%)]$ and vaginal discharge $[n=2(11 \%)]$. There was 1 case of premature labor (delivery at 34 weeks gestation). In total, 4 women experienced $\geq 1$ serious $\mathrm{AE}$ (SAE). The SAEs included blurred vision, sepsis, chorioamnionitis, intrauterine death, preeclampsia, and premature labor [each in 1 subject except chorioamnionitis $(n=2)$ ]; none were considered by the investigator to be at least possibly related to the study medication. One woman experienced chorioamnionitis associated with sepsis and intrauterine death of the fetus (all grade 3 in severity); this woman was withdrawn from the study due to terminated pregnancy.

Serum albumin and $\alpha_{1}$-acid glycoprotein concentrations were evaluated in 19 women at baseline, 15 at the second trimester visit, 13 at the third trimester visit, and 12 at the 612 weeks postpartum visit. Mean albumin

Table 3 Summary of AEs

\begin{tabular}{ll}
\hline Incidence, $\boldsymbol{n}(\%)$ & $\boldsymbol{n}=\mathbf{1 9}$ \\
\hline Any AE & $9(47)$ \\
Any AE considered at least possibly related to & 0 \\
$\quad$ study medication & 0 \\
Any AE leading to discontinuation & $4(21)$ \\
Any SAE & $1(5)$ \\
Any grade 3 or 4 AE & \\
Most common AEs (occurring in $>1$ woman) & \\
Chorioamnionitis & $2(16)$ \\
Vaginal discharge & $2(11)$ \\
\hline
\end{tabular}

$A E$ adverse event, $S A E$ serious adverse event 
concentrations were $32.8 \mathrm{~g} / \mathrm{L}$ (baseline), $31.6 \mathrm{~g} /$ $\mathrm{L}$ (second trimester), $29.6 \mathrm{~g} / \mathrm{L}$ (third trimester), and $39.0 \mathrm{~g} / \mathrm{L}$ (6-12 weeks postpartum). Mean $\alpha_{1^{-}}$ acid glycoprotein concentrations were $605.8 \mathrm{mg} / \mathrm{L}$ (baseline), $600.0 \mathrm{mg} / \mathrm{L}$ (second trimester), $603.1 \mathrm{mg} / \mathrm{L}$ (third trimester), and $955.0 \mathrm{mg} / \mathrm{L}$ (6-12 weeks postpartum).

Seven of the 12 infants (58\%) born to women who completed the study experienced $\geq 1 \mathrm{AE}$, all of which were grade 1 or 2 in severity; none of the AEs were considered by the investigator to be related to study medication or HIV infection. The most common AEs (occurring in $>1$ infant) were exomphalos $[n=2(17 \%)]$ and neonatal vomiting $[n=2(17 \%)]$. Six $(50 \%)$ infants experienced $\geq 1 \mathrm{SAE}$. These SAEs included talipes, ventricular septal defect, neonatal vomiting, neonatal fever, neonatal sepsis, medical observation, premature baby, and neonatal respiratory distress syndrome; each was reported in a single infant, except neonatal vomiting $[n=2(17 \%)]$, and none were considered by the investigator to be related to HIV infection.

\section{DISCUSSION}

Findings from the present study examining ARV pharmacokinetics in HIV-1-infected women demonstrated that exposure to rilpivirine is reduced during pregnancy compared with postpartum. Rilpivirine exposure was similar during the second and third trimesters of pregnancy. Compared with total rilpivirine, the reduction in exposure during pregnancy was less pronounced for unbound (pharmacodynamically active) rilpivirine. Despite the decrease during pregnancy, total rilpivirine $\mathrm{AUC}_{24 \mathrm{~h}}$ during each of the 3 time points (second and third trimesters of pregnancy and postpartum) was in the range of those observed in nonpregnant adults administered rilpivirine $25 \mathrm{mg}$ qd (mean \pm standard deviation $2235 \pm 851 \mathrm{ng} \cdot \mathrm{h} / \mathrm{mL}$ ) [13]. Similar decreases in total rilpivirine exposure during pregnancy have also been observed previously, including one study in which rilpivirine exposure (AUC) was $23 \%$ lower during the second trimester of pregnancy compared with postpartum and $20 \%$ lower during the third trimester of pregnancy compared with postpartum $[11,12]$. Apart from general physiologic changes that occur during pregnancy, these decreases in rilpivirine exposure during pregnancy are likely, at least partly, related to the metabolism of rilpivirine by the cytochrome P450 enzyme CYP34A, as the activity of this enzyme is increased during pregnancy $[1,3,13]$.

The earlier evaluations of rilpivirine pharmacokinetics during pregnancy focused only on total rilpivirine $[11,12]$. In the current study, which included assessment of unbound (pharmacodynamically active) rilpivirine, the decreases in exposure seen during pregnancy were less pronounced for unbound rilpivirine compared with total rilpivirine, although the differences were limited. Rilpivirine is approximately $99.7 \%$ bound to plasma proteins, primarily albumin [13]; however, changes in plasma protein content during pregnancy only had a limited impact on the fraction of unbound rilpivirine.

Rilpivirine had a favorable safety/tolerability profile in pregnant women; none of the maternal AEs were considered by the investigator to be at least possibly related to study medication and there were no discontinuations due to an AE. Importantly, despite decreased rilpivirine exposure during pregnancy, treatment was effective in suppressing HIV-1 infection in pregnant women and preventing mother-tochild transmission. No perinatal virus transmission was observed for any of the 10 infants with available data, consistent with previous studies [11, 12]. Individual cord/maternal plasma ratios of total rilpivirine on the day of delivery were also in the range of previous observations [median (range) 0.55 (0.3-0.8)] [12]. Moreover, rilpivirine was generally safe and well tolerated in infants; all infant AEs were mild in severity and none were considered by the investigator to be related to study medication or HIV infection. These results are similar to those of previous clinical studies assessing rilpivirine exposure in pregnant women.

This study was limited in some ways. The population size was small and data collection began after the first trimester of pregnancy. In addition, study medication administration was 
only observed on days in which women visited the clinic, and thus adherence during the study may have been incomplete for some women.

\section{CONCLUSION}

In summary, these study findings demonstrated that rilpivirine exposure was lower during pregnancy compared with postpartum, and the decrease was less pronounced for unbound rilpivirine compared with total rilpivirine. Despite this decrease in exposure, treatment with rilpivirine $25 \mathrm{mg}$ qd was effective in preventing mother-to-child virus transmission and in suppressing HIV-1 RNA in pregnant women in this study. Rilpivirine was generally safe and well tolerated in both women and their infants during pregnancy. Similar findings have been reported in other studies with rilpivirine in pregnant women $[11,12]$. Together, these results suggest that rilpivirine $25 \mathrm{mg}$ qd, as part of individualized cART, may be an appropriate option for HIV-1-infected pregnant women with close virologic monitoring.

\section{ACKNOWLEDGEMENTS}

The authors thank the patients and their families, and the study investigators.

Funding. Funding for this study and the article processing charges were provided by Janssen Scientific Affairs. All authors had full access to the study data and take responsibility for the integrity of the data and accuracy of the data analysis.

Medical Writing and/or Editorial Assistance. Medical writing support was provided by Courtney St. Amour, PhD, of MedErgy, and was funded by Janssen Scientific Affairs.

Authorship. All authors meet the International Committee of Medical Journal Editors (ICMJE) criteria for authorship for this manuscript, take responsibility for the integrity of the work as a whole, and have given final approval for the version to be published.
Prior Presentation. These data were previously presented, in part, in abstract and poster form at the 7th International Workshop on HIV \& Women, February 11-12, 2017, Seattle, WA, USA.

Disclosures. Olayemi Osiyemi has participated in speaker programs for Janssen, AbbVie, and Gilead; served on advisory boards for Bristol-Myers Squibb, Gilead, AbbVie, Janssen, and Durata; and received research grants from Forest Laboratories and Gilead. Salih Yasin has no financial interests to disclose. Carmen Zorrilla has received grant/research support from GlaxoSmithKline, Gilead, Merck, and Tibotec. Ceyhun Bicer is a consultant for Janssen and an employee of BICER Consulting \& Research (Antwerp, Belgium). Vera Hillewaert is a fulltime employee of Janssen. Kimberley Brown is a full-time employee of Janssen. Herta M. Crauwels is a full-time employee of Janssen.

Compliance with Ethics Guidelines. All subjects provided written informed consent to participate in this study. The study was conducted in accordance with the ethical principles that have their origin in the 1964 Declaration of Helsinki, and its later amendments, and is consistent with Good Clinical Practices and applicable regulatory requirements. The study protocol and amendments were reviewed by an independent ethics committee or institutional review board.

Data Availability. The datasets generated during and/or analyzed during the current study are available from the corresponding author on reasonable request.

Antiretroviral Pregnancy Registry Advisory Committee Consensus Statement. In reviewing all reported defects from the prospective registry, informed by clinical studies and retrospective reports of antiretroviral exposure, the Registry finds no apparent increases in frequency of specific defects with first trimester exposures and no pattern to suggest a common cause. While the Registry population exposed and monitored to date is not sufficient to detect an increase in the risk of relatively rare defects, 
these findings should provide some assurance when counseling patients. However, potential limitations of registries such as this should be recognized. The Registry is ongoing. Given the emergence of new therapies about which data are still insufficient, health care providers are strongly encouraged to report eligible patients to the Registry at http://www.APRegistry.com.

Open Access. This article is distributed under the terms of the Creative Commons Attribution-NonCommercial 4.0 International License (http://creativecommons.org/licenses/ by-nc/4.0/), which permits any noncommercial use, distribution, and reproduction in any medium, provided you give appropriate credit to the original author(s) and the source, provide a link to the Creative Commons license, and indicate if changes were made.

\section{REFERENCES}

1. Panel on Antiretroviral Guidelines for Adults and Adolescents. Guidelines for the use of antiretroviral agents in adults and adolescents living with HIV. Department of Health and Human Services. https:// aidsinfo.nih.gov/contentfiles/lvguidelines/Adultand AdolescentGL.pdf. Accessed 17 Oct 2017.

2. Panel on Treatment of Pregnant Women with HIV Infection and Prevention of Perinatal Transmission. Recommendations for use of antiretroviral drugs in pregnant women with HIV infection and interventions to reduce perinatal HIV transmission in the United States. https://aidsinfo.nih.gov/ contentfiles/lvguidelines/PerinatalGL.pdf. Accessed 30 Nov 2017.

3. Anderson GD. Pregnancy-induced changes in pharmacokinetics: a mechanistic-based approach. Clin Pharmacokinet. 2005;44(10):989-1008.

4. Gilbert EM, Darin KM, Scarsi KK, et al. Antiretroviral pharmacokinetics in pregnant women. Pharmacotherapy. 2015;35(9):838-55.

5. Zorrilla CD, Wright R, Osiyemi OO, et al. Total and unbound darunavir pharmacokinetics in pregnant women infected with HIV-1: results of a study of darunavir/ritonavir 600/100 mg administered twice daily. HIV Med. 2014;15(1):50-6.

6. Crauwels HM, Kakuda TN, Ryan B, et al. Pharmacokinetics of once-daily darunavir/ritonavir in HIV- 1-infected pregnant women. HIV Med. 2016;17(9):643-52.

7. Lamorde M, Byakika-Kibwika P, Okaba-Kayom V, et al. Suboptimal nevirapine steady-state pharmacokinetics during intrapartum compared with postpartum in HIV-1-seropositive Ugandan women. J Acquir Immune Defic Syndr. 2010;55(3):345-50.

8. Cressey TR, Stek A, Capparelli E, et al. Efavirenz pharmacokinetics during the third trimester of pregnancy and postpartum. J Acquir Immune Defic Syndr. 2012;59(3):245-52.

9. Ramgopal M, Osiyemi O, Zorrilla C, et al. Pharmacokinetics of total and unbound etravirine in HIV1-infected pregnant women. J Acquir Immune Defic Syndr. 2016;73(3):268-74.

10. Mulligan N, Schalkwijk S, Best BM, et al. Etravirine pharmacokinetics in HIV-infected pregnant women. Front Pharmacol. 2016;7:239.

11. Schalkwijk S, Colbers A, Konopnicki D, et al. Lowered rilpivirine exposure during the third trimester of pregnancy in human immunodeficiency virus type 1-infected women. Clin Infect Dis. 2017;65(8):1335-41.

12. Tran AH, Best BM, Stek A, et al. Pharmacokinetics of rilpivirine in HIV-infected pregnant women. J Acquir Immune Defic Syndr. 2016;72(3):289-96.

13. EDURANT $^{\circledR}$ (rilpivirine) [package insert]. Titusville: Janssen Therapeutics; 2015.

14. Cohen CJ, Molina JM, Cahn P, et al. Efficacy and safety of rilpivirine (TMC278) versus efavirenz at 48 weeks in treatment-naive HIV-1-infected patients: pooled results from the phase 3 doubleblind randomized ECHO and THRIVE Trials. J Acquir Immune Defic Syndr. 2012;60(1):33-42.

15. Nelson MR, Elion RA, Cohen CJ, et al. Rilpivirine versus efavirenz in HIV-1-infected subjects receiving emtricitabine/tenofovir DF: pooled 96-week data from ECHO and THRIVE studies. HIV Clin Trials. 2013;14(3):81-91.

16. Palella FJ Jr, Fisher M, Tebas P, et al. Simplification to rilpivirine/emtricitabine/tenofovir disoproxil fumarate from ritonavir-boosted protease inhibitor antiretroviral therapy in a randomized trial of HIV1 RNA-suppressed participants. AIDS. 2014;28(3):335-44.

17. Orkin C, DeJesus E, Ramgopal M, et al. Switching from tenofovir disoproxil fumarate to tenofovir alafenamide coformulated with rilpivirine and emtricitabine in virally suppressed adults with HIV1 infection: a randomised, double-blind, 
multicentre, phase $3 \mathrm{~b}$, non-interfority study. Lancet HIV. 2017;4(5):e195-204.

18. DeJesus E, Ramgopal M, Crofoot G, et al. Switching from efavirenz, emtricitabine, and tenofovir disproxil fumarate to tenofovir alafenamide coformulated with rilpivirine and emtricitabine in virally suppressed adults with HIV-1 infection: a randomised, double-blind, multicentre, phase $3 \mathrm{~b}$, noninferiority study. Lancet HIV. 2017. https://doi.org/ 10.1016/s2352-3018(17)30032-2 (Epub ahead of print).

19. European AIDS Clinical Society. EACS guidelines, version 9.0. 2017.

20. Antiretroviral Pregnancy Registry Steering Committee. Antiretroviral Pregnancy Registry
International Interim Report for 1 January 1989 through 31 January 2017. Wilmington: Registry Coordinating Center; 2017.

21. COMPLERA $^{\circledR}$ (emtricitabine, rilpivirine, tenofovir disoproxil fumarate) [package insert]. Foster City: Gilead Sciences; 2016.

22. Towers CV, Rumney PJ, Ghamsary MG. Longitudinal study of CD4+ cell counts in HIV-negative pregnant patients. J Matern Fetal Neonatal Med. 2010;23(10):1091-6.

23. Notarianni LJ. Plasma protein binding of drugs in pregnancy and in neonates. Clin Pharmacokinet. 1990;18(1):20-36. 